\title{
Research on Construction of Agricultural Domain Knowledge Service Platform Based on Ontology
}

\author{
Yuantao Kou ${ }^{1, \mathrm{a}}$, Ruixue Zhao ${ }^{1, \mathrm{~b}}$, Guojian Xian ${ }^{1, \mathrm{c}}$ \\ ${ }^{1}$ Agricultural Information Institute, Chinese Academy of Agricultural Sciences,Beijing \\ 100081, China \\ akouyuantao@caas.cn, bhaoruixue@caas.cn, cxianguojian@caas.cn
}

\begin{abstract}
Scientific researchers' increasing demand for knowledge service under the new situation, makes it urgent to embed information service into user research process, ad build an incorporate knowledge platform that integrates knowledge, skills, tools, and services of certain professional field. This paper put forward the technical solution of agricultural domain knowledge service platform based on ontology, including resource organization based on ontology, platform design and development. The construction progress of ontology base and service functions based on ontology are shown by application practice in rice domain.
\end{abstract}

Keywords: domain knowledge service, knowledge organization system, scientific research ontology, domain ontology

\section{$1 \quad$ Introduction}

Scientific and technological innovation is the source of power that promotes social development and progress. In the era of knowledge economy, innovation and application of knowledge has become the most important part of scientific and technological innovation. Any research on scientific and technological innovation is inseparable from the support of science and technology information resources, and the construction of e-science environment.

Scientific users have an increasing needs [1,2] for service integration, knowledge management, knowledge services, communication and collaboration under the new situation. This makes it become urgent to build a research and knowledge service 
platform to integrate knowledge, technology, tools, services of certain domain, so as to embed information services into users' research process, achieve service innovation, and support the scientific and technological innovation.

Research on the construction of domain knowledge service platform has made significant progress internationally. Lots of countries are paying more and more attention to domain knowledge service platform, and some large, national level research projects and practical activities have been carried up. There are some systems or software tools that we can refer to, such as VIVO based on ontology [3,4], VRE based on SOA [5,6], Harvard Catalyst, Sciologer of Columbia University [7], SKE of CAS [8], the open source software of virtual learning environment SaKai, [9-11], etc. These construction experiences provide a good foundation for this study.

Ontology is a good technical tool which shares common understanding of the structure of information among people or software agents and enables reuse of domain knowledge. This paper put forward the technical solution of agricultural domain knowledge service platform based on ontology, including resource organization based on ontology and platform design and development. Current construction and application situation in rice domain was introduced at the end.

\section{Resource organization based on ontology}

Resources organization is the root of a knowledge service system. Knowledge organization system construction is foundation and core of realizing the transformation from scientific literature information services to knowledge service. Agriculture itself is a complex subject, so what needs to be solved in this system is the organization of core knowledge content. Using ontology which can represent the meaning and relevance of knowledge more accurately, laid the foundation for construction of the knowledge service application.

Regarding domain knowledge service application as the guidance, advanced international mainstream knowledge organization technology should be adopted in agricultural domain knowledge organization. Existing knowledge organization materials were used for reference in ontology design and ontology instance base construction. Ontology of agricultural domain knowledge service platform consists of scientific research ontology and domain ontology. 


\subsection{Scientific research ontology}

Scientific research ontology reveals and reflects research elements and their mutual connection, which is the foundation of knowledge discovery. VIVO, put forward by Connell University, is very useful reference. VIVO integrated with powerful ontology management tools, through construction of ontology around scientific experts ("people"), using Jena inference system to realize associated navigation and retrieval of research objects $[12,13]$. Considering scientific research agent, research condition, research activities and research output are core concepts during scientific research progress, in this paper, we proposed the scientific research ontology around research progress. Its main class resources are as Fig.1.

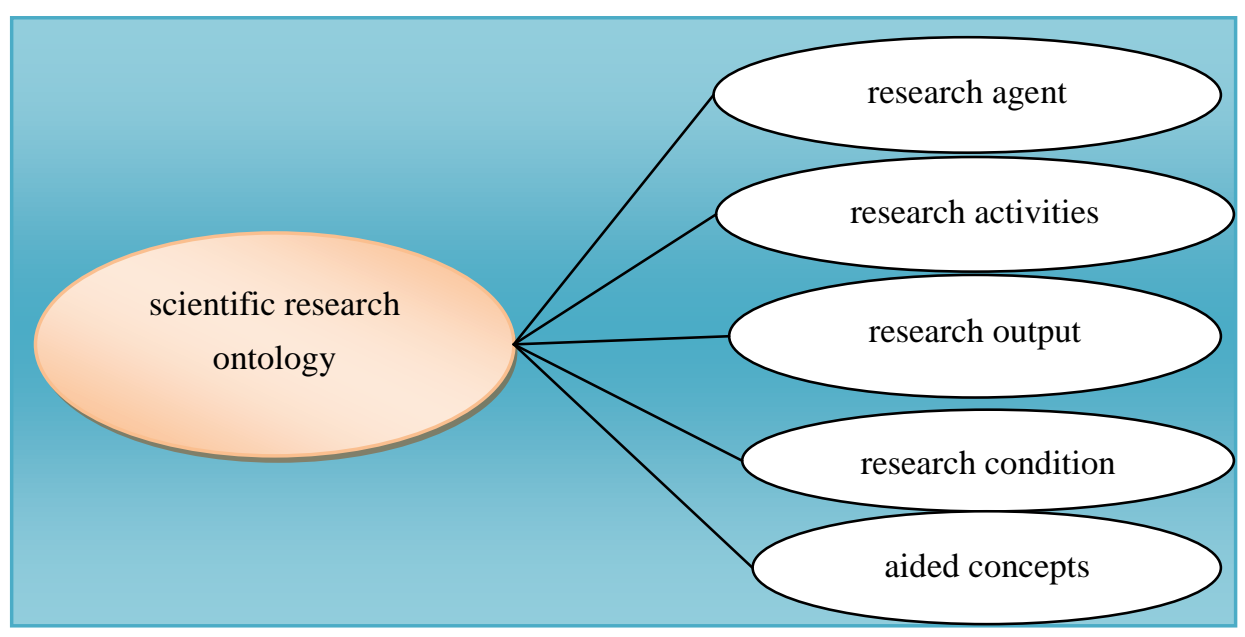

Fig. 1. Main class resources of scientific research ontology

Fig. 2 depicts the research output class. Its sub classes include journals, dissertations, proceedings, books, academic reports, scientific data, patents, and standards. The figure also shows the relationship between research output and other major classes through object properties. 


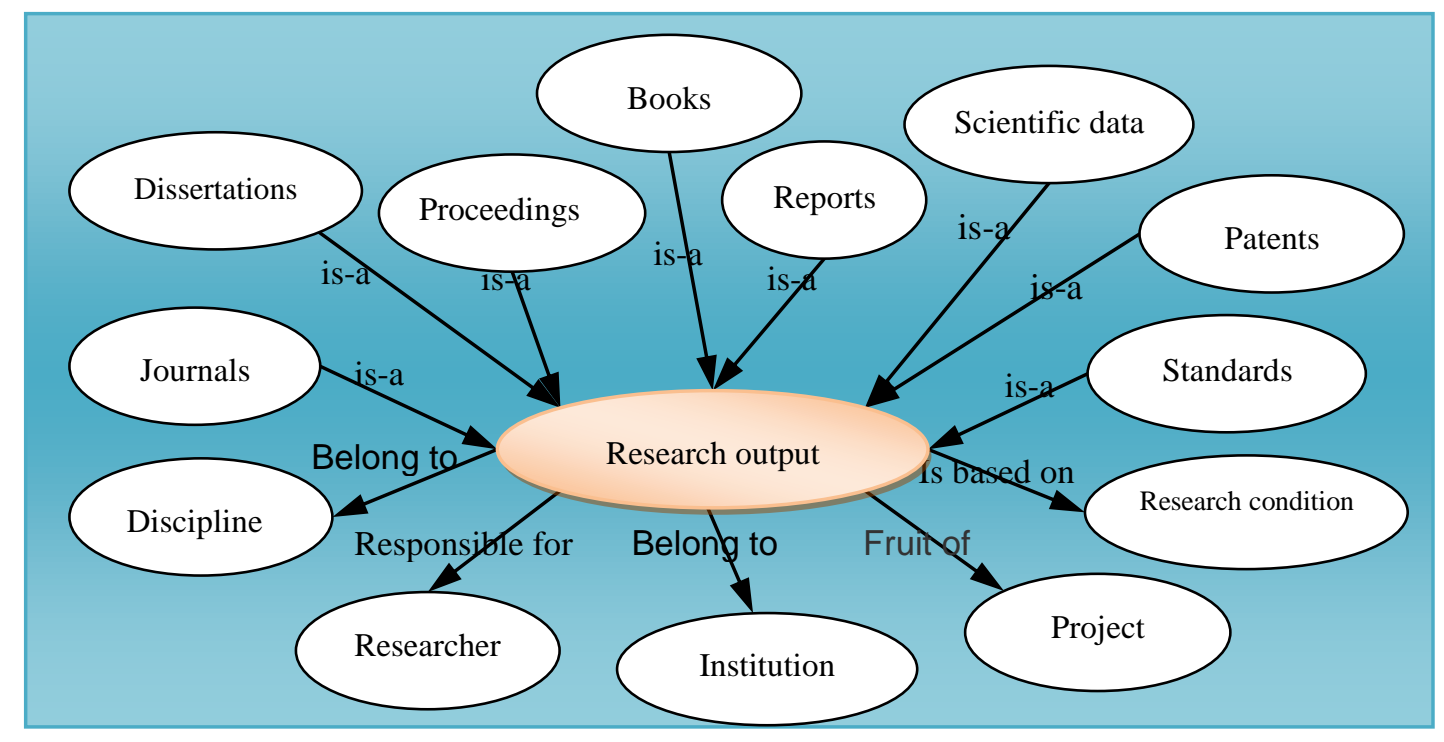

Fig. 2. Class diagram of scientific research output

\subsection{Domain ontology}

Domain ontology is domain specific, and constructed on the basis of analysis of knowledge content and core concepts, their attributes and the relationship between them. And it's used for knowledge organization and knowledge association discovery of research objects and literature information, which are the base of domain knowledge navigation and knowledge structure exhibition.

Domain ontology can focus on the whole domain, or a small part of it, or even be a combination of several disciplines. According to the general ontology framework, extending ontology instances facing specific area is the key of content construction of agricultural domain knowledge service system. Therefore the domain ontology of this platform will be constructed after the target application domain is identified.

\section{Platform design and development}

Agricultural domain knowledge service platform is a personalized information platform for professional researchers, and also an integrated information system which is 
the organic integration of library services, users' research process and the internet service environment.

\subsection{System architecture}

This paper presents the 4-layer architecture of agricultural domain knowledge service platform, as shown in Fig.3, which includes support layer, resource layer, application layer and user layer.

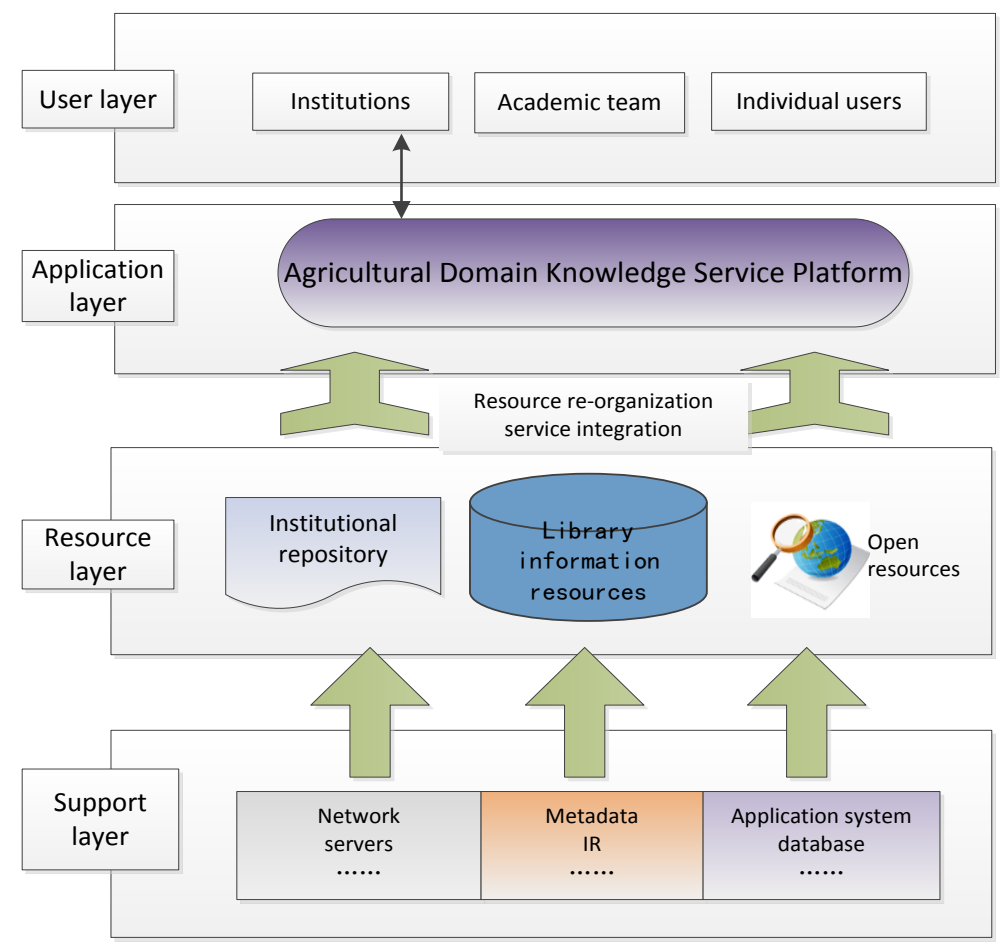

Fig. 3. System architecture

- The user layer is located at the top level of the whole structure, and gives out the primary users of agricultural domain knowledge service platform, including research users, manage users and third party users. Scientific users are the main service target which is made up with researchers, research teams and research institutions. 
- The application layer, which mainly aims to solve the problem of platform's construction, realizes various services of agricultural domain knowledge service platform in the way of system functions and tools with seamless integration of third-party technology tools from existing service platform or open source systems.

- The resource layer, which is responsible for resource organization, clearly defines the resources elements of domain knowledge service platform, carries out the collection and procession of the resources, and builds the domain knowledge base using ontology.

- The underlying support layer aims at the building of hardware and software environment which is the technical foundation of the entire system.

\subsection{Functional structure}

Fig. 4 shows the functional structure of agricultural domain knowledge service platform, and the green parts included are its main components:

- Knowledge service functions mainly provide the core services of agricultural domain knowledge service platform, including domain knowledge navigation, knowledge retrieval and acquisition, domain knowledge association, knowledge maps, knowledge sharing, knowledge management and other functional components.

- The domain knowledge organization and management tools mainly achieve content construction of agricultural domain knowledge service platform, maintenance of classes and attributes of scientific research ontology and domain ontology, as well as the processing and transformation of resource materials to database.

- Collaborative modules which mainly support researchers' tacit knowledge sharing and provide online communication and knowledge sharing environment, include collaborative work platform, scientific community, domain knowledge wiki, scholars' network and other functions.

- Data service interface mainly achieves the scalability and extensibility of agricultural domain knowledge service platform, supporting data exchange between the platform and third-party systems or services.

- The system basic functions such as system management, user and privilege management, and statistics support the system's normal operation. 


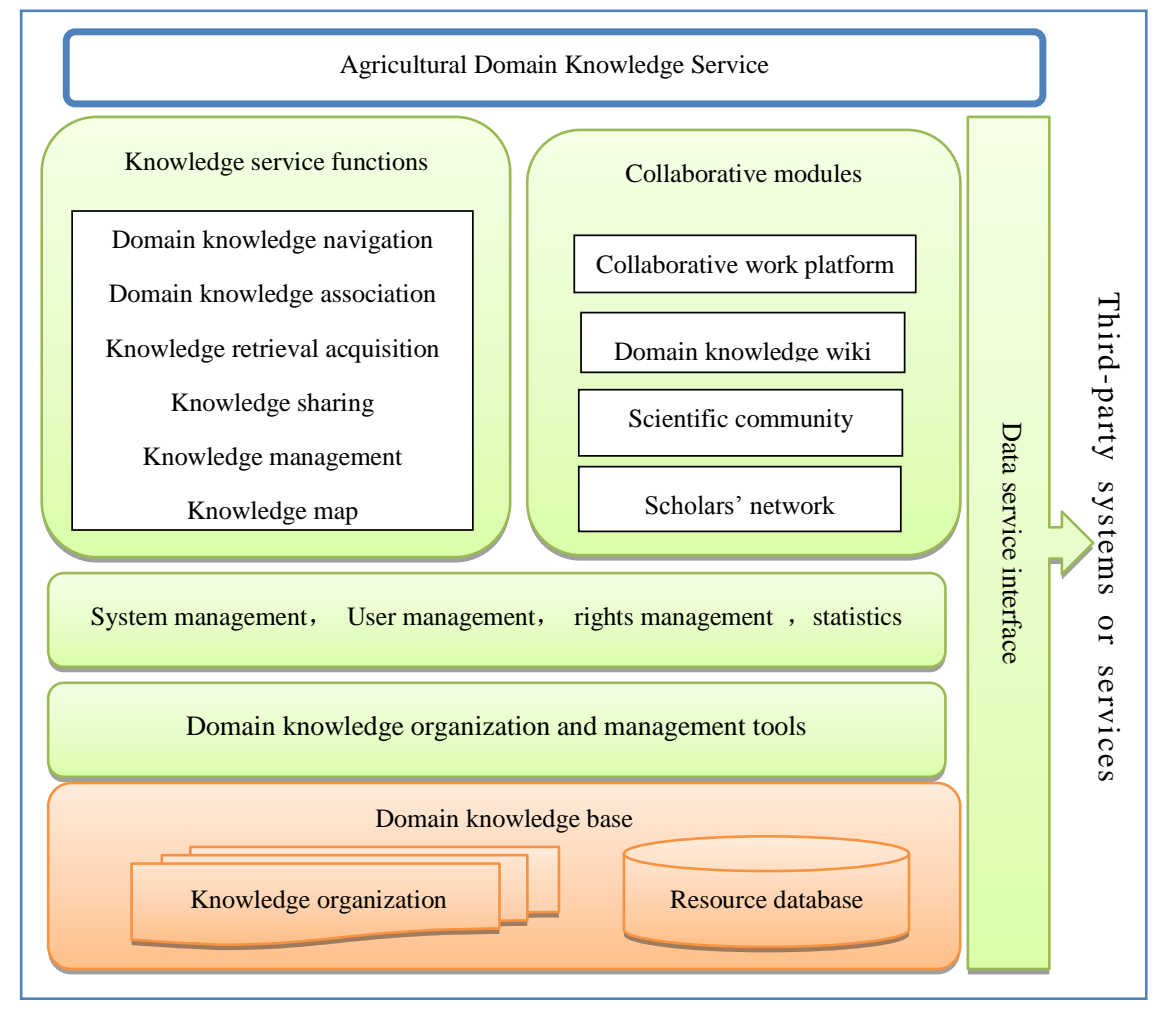

Fig. 4. Functional structure of Agricultural domain knowledge service platform

\subsection{Platform development}

According to the characteristics of large volume data of the system, distributed architecture was used, so as to benefit sharing system computation and storage pressure. Java was the main development language, based on J2EE architecture, and MyEclipse was the development tool, with the environment of "Windows2008+ Tomcat6.0+Mysq15.5".

During construction of the knowledge base, O-R (ontology relation) mapping technique was applied to achieve dynamic storage of resources, and Hessian technology was used to obtain the cross language, cross platform and more openness of the knowledge base.

By adopting the SSH structure, knowledge application of the foreground system could dynamically adjust columns and information project from the knowledge structure, to support cross domain application. Knowledge service functions were realized by us- 
ing AJAX, RSS, GIS, knowledge visualization and other related software technology. And knowledge organization and management functions in background system were implemented by "generic desktop" technology, which provides Windows operating experience under the Web system.

\section{Application practice in rice domain}

Application to a specific domain is the "last mile" to realize construction target of agricultural domain knowledge service platform. Combined with country-level major projects, according to the discipline construction and development status of Chinese academy of Agricultural Sciences, we selected rice as the target domain and deployed the rice domain knowledge service system, which provides knowledge service to scientific workers engaged in rice research. Fig.5 gives the rice knowledge service system homepage screenshots.

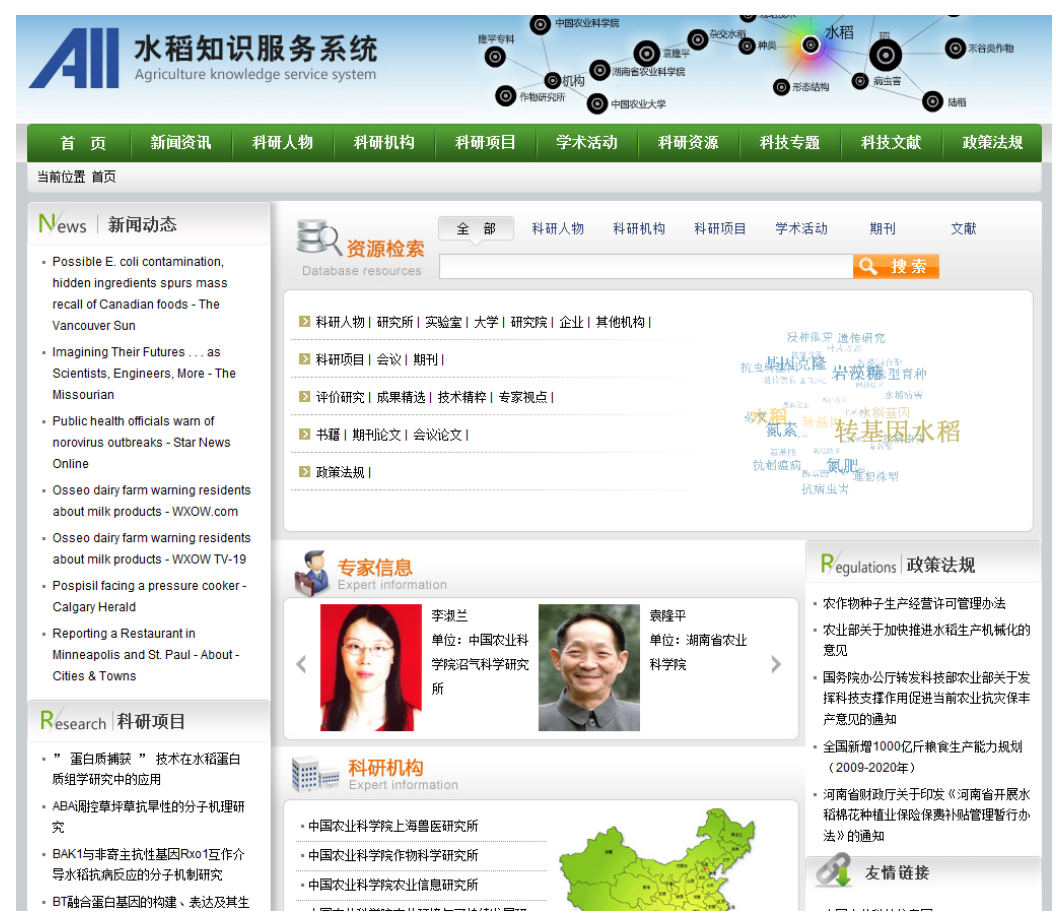


Fig. 5. Rice knowledge service system homepage

\subsection{Ontology base construction}

As to scientific research ontology base of rice domain, we carried out collecting, processing and arranging information resources about rice, including scientific research institutions, scientific research personnel, scientific literature, science data, knowledge organization system, etc.

Rice domain knowledge is extremely large and rich, covering rice varieties, rice cultivation, rice producing areas, rice biochemistry, rice diseases and insect pests, weeds and so on. So it's quite difficult to describe the knowledge content in detail for the whole rice domain with ontology. In view of this, this paper selected the sub field --plant diseases and insect pests of rice, as the research object of lightweight domain ontology construction.

Rice plant diseases and insect pests ontology mainly describes the concepts about symptom, pathogen, occurrence of damage, occurrence time, occurrence conditions, route of transmission, and prevention and control of rice disease and insect pests, as well as the relationships between these concepts. The construction method is, on the basis of professional books and database resources, analyze the knowledge content structure, disjunctive core concepts and relations, extract the pest discipline branches, complete the design of ontology framework, and finally complete the construction in protégé.

\subsection{Service functions based on ontology}

In rice domain knowledge service system, besides the ordinary functions including domain knowledge retrieval, knowledge navigation, knowledge acquisition, and maintenance of knowledge organization system, there are two typical service functions based on ontology.

First is visualization of research entities and their relationships. As shown in Fig.6, scientific researchers, research topics, institutions, literature, and relationship between them are revealed in one "knowledge map". 


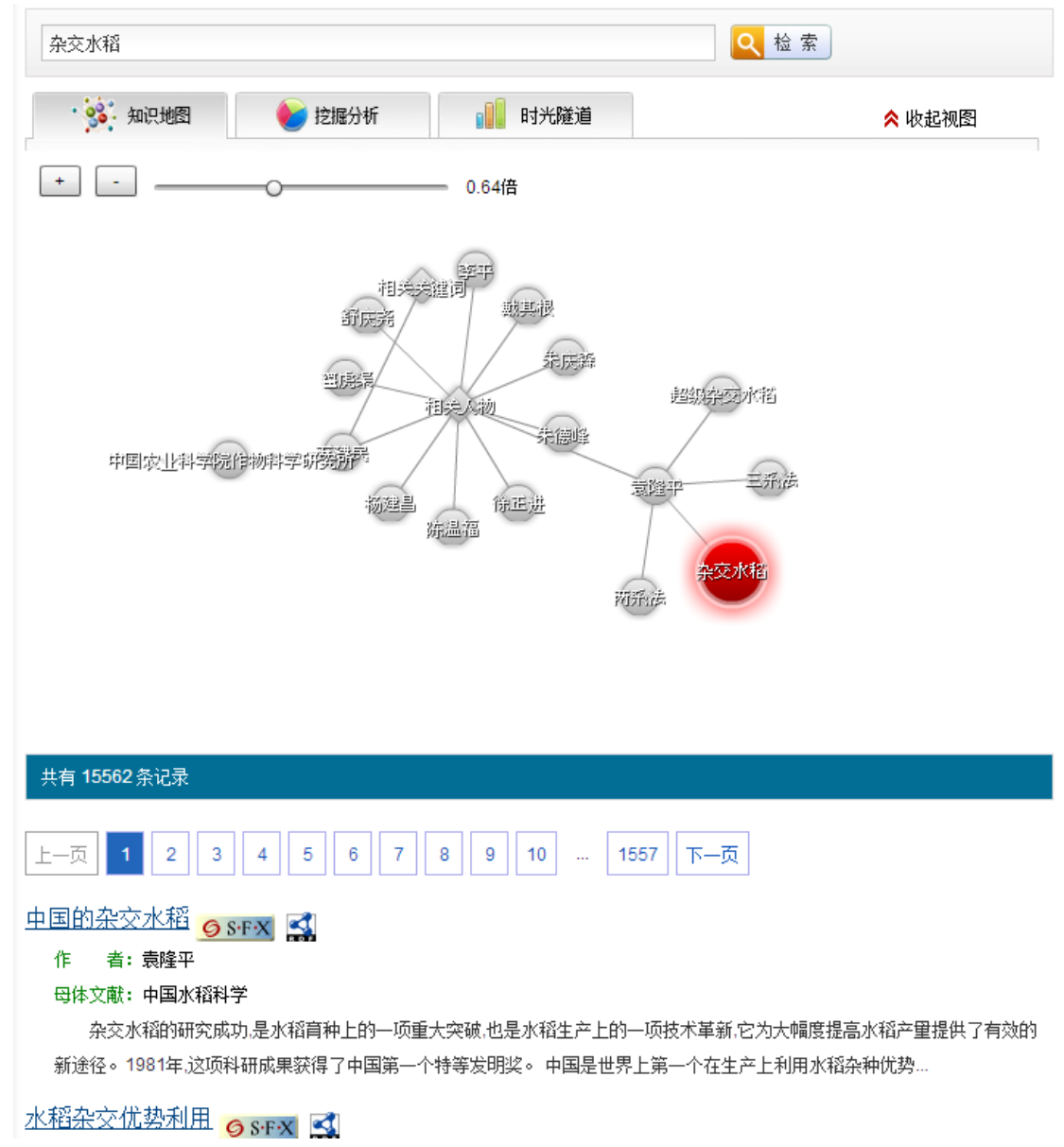

Fig. 6. Visualization of research entities and their relationships

Secondly, based on the semantic inference of domain ontology, rice plant diseases and insect pests diagnosis reasoning was realized. There are two forms of diagnosis in the platform: one is that users input the names of plant diseases and insect pests, and then the platform provides basic information of the disease or insect pest, as well as related symptoms, occurrence position, rice growth period, methods of prevention, drugs, and relevant literature; another is that, with some symptoms input by users, the platform presents suspected plant diseases and insect pests after reasoning based on ontology, so as to give treatment suggestion and relevant literature links (Fig.7). 


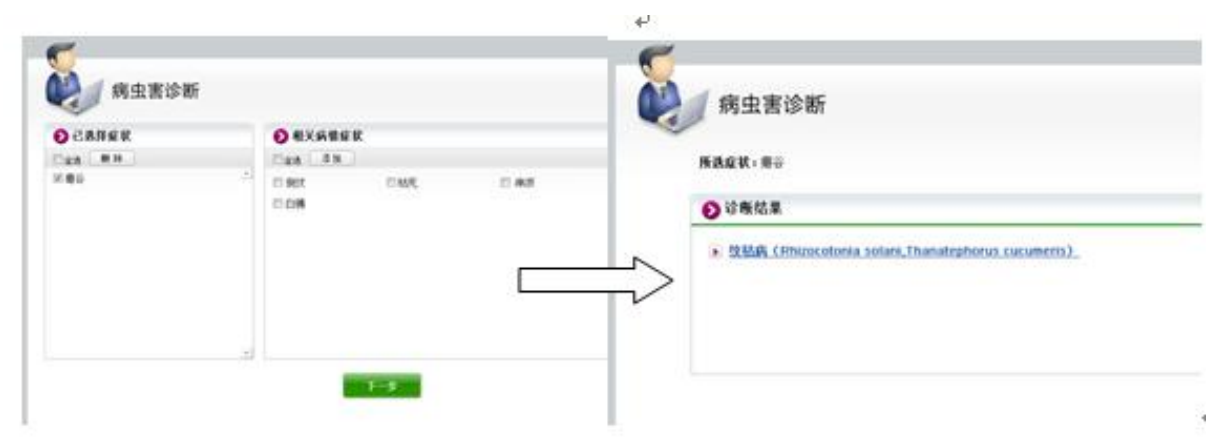

Fig. 7. Rice plant diseases and insect pests diagnosis

\section{Conclusion}

Domain knowledge service platform is not a new service model, but its construction is still in the exploratory. The practice we have tried using ontology in agriculture domain knowledge service is very meaningful. However, there are still a lot of issues that need further improvement and solvent in the future, such as core services, construction of domain ontology, data standards, users' research process and their work environment, non-technical factors, interdisciplinary knowledge exchange and so on. In short, building an integrated knowledge service platform for professional areas is a general trend, but carrying out the work in a large scale and showing the effectiveness still need a period of time. That needs constant exploration to new technologies, new methods of knowledge services to continuously promote technology innovation.

\section{Acknowledgement}

The related research work of this paper was support by the National Science \& Technology Pillar Program "Knowledge Services Application and Demonstration Based on STKOS" (Grant No. 2011BAH10B06) during the Twelfth Five-year Plan Period of China. 


\section{References}

1. Xiao Xiantao, Wang Dandan. Development trend of user information environment, information behavior and information demand [J]. Library Theory and Practice, 2010(1):40-42(in Chinese).

2. Chen Chengxin. Users Information Requirements and Information Services under E-science Environment [J]. Information Science,2009, 27(1):108-112(in Chinese).

3. Dean B. Krafft, eduNicholas A. Cappadona, Brian Caruso, etc. VIVO: Enabling National Networking of Scientists[C]. Web Science Conf. 2010, April 26-27, 2010, Raleigh, NC, USA.

4. Medha Devare, Jon Corson-Rikert, Brian Caruso, etal. VIVO Connecting People, Creating a Virtual Life Sciences Community[J]. D-Lib Magazine, 2007, 13(7/8) .

5. Rob Allan,Alison Allden, David Boyd,etal. Roadmap for a UK Virtual Research Environment. Report of the JCSR VRE Working Group[R], 2004.

6. Xiaobo Yang, Rob Allan. Web-Based Virtual Research Environments (VRE): Support Collaboration in e-Science[C]. Proceedings of the 2006 IEEE/WIC/ACM international conference on Web Intelligence and Intelligent Agent Technology. Washington, DC, USA : IEEE Computer Society, 2006:p184-187.

7. Comparison with VIVO. [EB/OL]. (January 23, 2012).

http://swl.slis.indiana.edu/repository/systemdoc_files/comparison_with_VIVO\%2012 -01.pdf.

8. Song Wen, Zhang Shinan. Ideas and Applications of Subject Knowledge Environments[J]. Library Theory and Practice, 2012(1):30-33(in Chinese).

9. Borda, A., Careless, J., \& Dimitrova, M., et al. Report of the working group on virtual researchcommunities for the OST e-infrastructure steering group. London, UK: Office of Science andTechnology, 2006: 33-36.

10. Allan, R., Allden, A., \& Boyd, D., et al. Roadmap for a UK virtual research environment.Report of the JCSR VRE working group, 2004.

11. Yang, X.B., \& Allan, R. Web-based virtual research environments (VRE): Support collaborationin e-Science, In Butz, C., Nguyen, N.T., \& Takama, Y.(Eds.): Proceedings of the 2006 IEEE/WIC/ACM international conference on Web Intelligence and intelligent agent technology.Washington, DC, USA: IEEE Computer Society, 2006: 184-187. 
12. KRAFFT D B, CAPPADONA N A, CARUSO B, et al. VIVO: Enabling National Networking of Scientists[C]// Web Science Conf. 2010, April 26-27, 2010, Raleigh,NC, USA.

13. DEVARE M, CORSON-RIKERT J, CARUSO B, et al. VIVO: Connecting People, Creating a Virtual Life Sciences Community[J]. D-Lib Magazine, 2007(13):7-8. 